\title{
Dissected Aorta Repair Through Stent Implantation trial: Canadian results
}

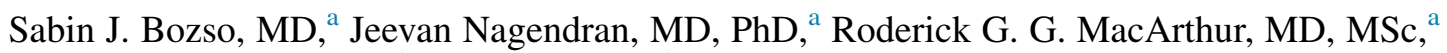
Michael W. A. Chu, MD, ${ }^{b}$ Bob Kiaii, MD, ${ }^{b}$ Ismail El-Hamamsy, MD, PhD, ${ }^{c}$ Raymond Cartier, MD, Ali Shahriari, MD, ${ }^{\mathrm{d}}$ and Michael C. Moon, $\mathrm{MD}^{\mathrm{a}}$

\section{ABSTRACT}

Objectives: We describe the Canadian results of the Ascyrus Medical Dissection Stent (AMDS), a novel partially uncovered aortic arch hybrid graft implanted antegrade during hypothermic circulatory arrest to promote true lumen expansion and enhance aortic remodeling.

Methods: From March 2017 to February 2018, 16 consecutive patients (66 \pm 12 years; $38 \%$ female) presented with acute type A aortic dissections and underwent emergent surgical aortic repair with AMDS implantation. All patients presented with DeBakey I aortic dissection, with evidence of malperfusion in $50 \%(\mathrm{n}=8)$ of patients. All cases were performed under hypothermic circulatory arrest with an additional average duration for AMDS implantation time of 2.1 minutes.

Results: All 16 device implantations were successful. Overall 30-day mortality was $6.3 \%(\mathrm{n}=1)$ and stroke occurred in $6.3 \%(\mathrm{n}=1)$ of cases. There was no incidence of device-related aortic injury or aortic arch branch vessel occlusion. During the follow-up period, 12 patients had completed at least 1 postoperative computed tomography scan. At initial follow-up computed tomography scan, complete or partial thrombosis, and remodeling of the aortic arch occurred in $91.7 \%$ of cases $(\mathrm{n}=11 / 12)$ and in the proximal descending thoracic aorta, complete or partial thrombosis, and remodeling occurred in $91.7 \%(\mathrm{n}=11 / 12)$.

Conclusions: Preliminary results suggest that the AMDS is a safe, feasible and reproducible adjunct to current surgical approaches for acute DeBakey I aortic dissection repair. Further, the AMDS manages malperfusion and promotes early positive remodeling in the aortic arch and distal dissected segments, with favorable FL closure rates at follow-up. Ongoing follow-up will provide additional insight into the long-term effects of the AMDS. (J Thorac Cardiovasc Surg 2019;157:1763-71)

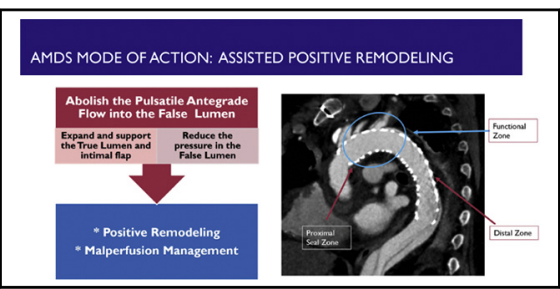

The AMDS mode of action in promoting positive aortic remodeling

Central Message

The AMDS is a safe, feasible, and reproducible adjunct to current surgical aortic dissection repair, and promotes early positive remodeling with favorable FL closure rates at follow-up.

\section{Perspective}

The AMDS is a safe, feasible, and reproducible adjunct to current surgical approaches for aortic dissection repair without prolonging the procedure. The early results indicate that the AMDS promotes early positive remodeling and might be an effective treatment for dynamic malperfusion involving major aortic branches without introducing new risks to the current standard of care hemiarch aortic reconstruction. Follow-up will provide insight into the long-term effects of the AMDS on aortic remodeling and patient outcomes.

See Editorial Commentary page 1772

\footnotetext{
From the a Division of Cardiac Surgery, University of Alberta, Edmonton, Alberta, Canada; 'bivision of Cardiac Surgery, Western University, London, Ontario, Canada; ${ }^{\mathrm{c}}$ Division of Cardiac Surgery, Montreal Heart Institute, Montreal, Quebec, Canada; and ${ }^{\mathrm{d}}$ Ascyrus Medical, LLC, Boca Raton, Fla.

Funding for this study was provided by Ascyrus Medical (Boca Raton, Fla).

Read at the 98th Annual Meeting of The American Association for Thoracic Surgery, San Diego, California, April 28-May 1, 2018.

Received for publication May 1, 2018; revisions received Aug 8, 2018; accepted for publication Sept 4, 2018; available ahead of print Nov 27, 2018.

Address for reprints: Michael C. Moon, MD, 4A8.082, Mazankowski Alberta Heart Institute, 8440112 St, Edmonton, Alberta T6G 2B7, Canada (E-mail: bozso@ ualberta.ca).

$0022-5223 / \$ 36.00$

Copyright (c) 2018 by The American Association for Thoracic Surgery

https://doi.org/10.1016/j.jtcvs.2018.09.120
}

Acute DeBakey I aortic dissection is a life-threatening condition that requires emergent repair. The most common surgical approach consists of resecting the primary entry tear and performing a hemiarch repair, often leaving residual untreated false lumen (FL) in the distal aorta. Although the standard hemiarch repair is often a life-saving measure, the inability to re-expand the true lumen (TL) and abolish

\begin{tabular}{|c|c|c|}
\hline & $\begin{array}{l}\text { Scanning this QR code will } \\
\text { take you to the article title } \\
\text { page to access supplementary } \\
\text { information. }\end{array}$ & 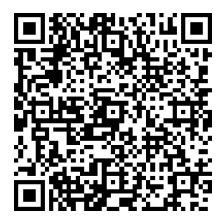 \\
\hline
\end{tabular}




$$
\begin{aligned}
& \text { Abbreviations and Acronyms } \\
& \text { AMDS }=\text { Ascyrus Medical Dissection Stent } \\
& \text { APF }=\text { antegrade pulsatile flow } \\
& \text { ASA }=\text { acetylsalicylic acid } \\
& \text { CT }=\text { computed tomography } \\
& \text { DARTS }=\text { Dissected Aorta Repair Through Stent } \\
& \text { Implantation } \\
& \text { FET }=\text { frozen elephant trunk } \\
& \text { FL }=\text { false lumen } \\
& \text { MSCT }=\text { multislice computed tomography } \\
& \text { PTFE }=\text { polytetrafluoroethylene } \\
& \text { TL }=\text { true lumen }
\end{aligned}
$$

the antegrade pulsatile flow (APF) into the FL causes ineffective treatment of malperfusion in the short term and contributes to negative remodeling in most patients, causing significant morbidity and mortality in the intermediate and long term. Patency of the FL after hemiarch repair of acute DeBakey I aortic dissection ranges from $69.7 \%$ to $78 \%{ }^{1-4}$ This leads to approximately $76 \%$ of patients with an untreated FL patency developing unfavorable remodeling. ${ }^{5}$ In fact, Rylski and colleagues ${ }^{5}$ showed that $96 \%$ of patients with negative remodeling and accelerated aortic growth had an entry tear and communication to the FL at the graft-aorta anastomosis. As a result of this unfavorable remodeling, approximately $40 \%$ to $50 \%$ of patients require further intervention on the aorta in the first decade after diagnosis and $50 \%$ are at risk of mortality. ${ }^{6,7}$ Consequently, between $45.6 \%$ and $50 \%$ of secondary aortic interventions target FL expansion and aneurysmal degeneration in the aortic arch. ${ }^{8,9}$ In the past, attempts have been made to relaminate the aorta and to reapproximate the intimal flap to the aortic wall to remedy the chronic expansion of the aortic arch. One device that was introduced for this purpose was the Djumbodis stent. ${ }^{10}$ The Djumbodis stent is an uncovered stainless steel balloon-expanding frame that was introduced into the TL during circulatory arrest and ballooned open to reapproximate the intimal flap to the aortic wall. The Djumbodis lacks a sealing component for the hemiarch anastomotic area; its design used force for relamination and the conformability of the stainless steel frame was challenged in the hostile arch anatomy leading to stent fractures.

The Ascyrus Medical Dissection Stent (AMDS) is a novel, partially uncovered aortic arch hybrid graft made of a proximal Teflon fabric graft sewn to a uniquely designed tubular Nitinol frame with a low chronic outward force, high kink resistance, and ability to conform to the curvature of the aortic arch. The AMDS provides long thoracic aortic coverage to alleviate malperfusion and a large open-cell structure to allow uninhibited perfusion of major aortic branches and re-intervention of aortic branch vessels, distal re-entry tears, and treatment directed to the FL if necessary. The AMDS has been developed as an adjunct to standard surgical repair and designed to improve malperfusion and promote positive remodeling of the aortic arch and distal dissected aorta at the time of initial surgery without significantly prolonging or complicating the standard of care repair in this gravely ill patient population. The aim of this article is to report the initial Canadian experience with the AMDS while highlighting the safety, feasibility, and performance of the device.

\section{METHODS \\ Trial Design}

The Dissected Aorta Repair Through Stent Implantation (DARTS; ClinicalTrials.gov Identifier: NCT03397251) trial was designed to analyze the safety, feasibility, and performance of the AMDS device. The DARTS trial is a multicenter, prospective, nonrandomized, nonblinded, single-arm design trial. Patients between 18 and 80 years of age who have been diagnosed with an acute DeBakey I aortic dissection or intramural hematoma within 14 days of the diagnosis were included. Patients were excluded if they had suffered a clinically detectable stroke within 30 days, were in extreme hemodynamic compromise requiring cardiopulmonary resuscitation, had an arch or proximal descending thoracic aortic aneurysm measuring $>45 \mathrm{~mm}$, or had a diagnosis of Marfan, Loeys-Dietz, or Ehlers-Danlos syndrome. No patients were excluded because of anatomical restrictions. The treatment of re-entry tears in the aortic arch were left to the discretion of the investigator as long as the primary entry tear in the ascending aorta was resected. The primary outcome measures are: 30-day mortality, neurologic dysfunction, aortic injury associated with device implantation, aortic branch vessel patency, and rates of positive aortic remodeling. Positive aortic remodeling was assessed using comprehensive multislice computed tomography (MSCT) imaging for all patients enrolled in the DARTS trial by a single individual. Positive remodeling was defined using internationally agreed upon criteria. ${ }^{11-13}$ These are on the basis of any of the following 3 anatomical criteria alone or in combination: FL obliteration, complete FL thrombosis, and favorable changes in aortic dimensions, including stable or decreased total aortic diameter $( \pm 5 \mathrm{~mm})$, stable or increased TL size $( \pm 5 \mathrm{~mm})$, and stable or decreased FL size $( \pm 5 \mathrm{~mm})$. In this trial, partial thrombosis of the FL in combination with positive dimensional changes of the aorta serves as a surrogate for AMDS-induced decrease in FL flow and positive remodeling. Although publications such as that by Tsai and colleagues ${ }^{14}$ have shown that patients with partial thrombosis of the untreated FL might show negative remodeling and accelerated growth, the group of patients with an untreated FL is not comparable with the DARTS population. Long-term follow-up of the patients in this trial will help further clarify the effect of complete and partial thrombosis on patient outcomes. Malperfusion was assessed clinically and radiographically on the immediate postoperative MSCT scans. Measurement of the size of the total aorta, TL, and FL was performed using 3-D postprocessing of the computed tomography (CT) angiograms using the Osirix Imaging system (Bernex, Switzerland). The total diameter of the aorta and greatest depth of the TL and FL was measured and the changes in the representative regions were measured and compared on follow-up CT scans.

\section{Patients}

From March 2017 to February 2018, a total of 16 patients presented with acute DeBakey I aortic dissections and underwent emergent surgical aortic repair with AMDS implantation. All procedures were performed at 3 institutions in Canada: the Mazankowski Alberta Heart Institute, University of 
TABLE 1. Baseline characteristics of the study population

\begin{tabular}{|c|c|}
\hline Characteristic & Value \\
\hline Age, y & $66 \pm 12$ \\
\hline Female sex & $37.5 \%(6 / 16)$ \\
\hline Total arch replacement & $6.3 \%(1 / 16)$ \\
\hline Malperfusion & $50 \%(8 / 16)$ \\
\hline Reoperation & $12.5 \%(2 / 16)$ \\
\hline Hypertension & $68.8 \%(11 / 16)$ \\
\hline Previous stroke & $12.5 \%(2 / 16)$ \\
\hline COPD & $18.8 \%(3 / 16)$ \\
\hline Chronic renal failure & $12.5 \%(2 / 16)$ \\
\hline Mean DHCA duration, minutes & $33 \pm 12$ \\
\hline Mean cerebral perfusion duration, minutes & $27.3 \pm 10.4$ \\
\hline Mean cross-clamp duration, minutes & $154 \pm 70$ \\
\hline Mean AMDS implantation time, minutes & 2.1 \\
\hline
\end{tabular}

COPD, Chronic obstructive pulmonary disease; $D H C A$, deep hypothermic circulatory arrest; $A M D S$, Ascyrus Medical Dissection Stent.

Alberta, in Edmonton, Alberta; the University Hospital, London Health Sciences Centre, Western University, in London, Ontario; and the Montreal Heart Institute, in Montreal, Quebec. Baseline characteristics of the study population and intraoperative technical details are shown in Table 1. The reasons for exclusion for individuals screened, but not enrolled in the study is provided in Table 2. Device safety outcomes and short-term clinical outcomes were evaluated and reported for the complete study population. All patients were treated through a research protocol approved by local research ethics boards and Health Canada.

\section{The AMDS Design}

The AMDS is a novel, partially uncovered aortic arch hybrid graft made of Teflon fabric graft sewn to a tubular Nitinol frame designed to be implanted antegrade during hypothermic circulatory arrest into the aortic arch and the descending thoracic aorta (Figures 1 and 2). The device consists of a felt sewing cuff proximally to facilitate the distal aortic anastomosis and a novel asymmetric superhelical stent frame design with low outward force to allow for TL expansion and accommodation of the curvature within the aortic arch without kinking and without exerting undue forces on the friable aorta. The stent component is designed to only expand and support the TL without attempting forceful relamination. The delivery system may be deployed with or without the use of a guide wire or fluoroscopy and facilitates a simple 1-step deployment and stable positioning

TABLE 2. Study exclusions

\begin{tabular}{lc}
\hline \multicolumn{1}{c}{ Variable } & Value \\
\hline Number of individuals screened & 28 \\
Number of individuals excluded & 12 \\
Reason for exclusion & \\
$\quad$ DeBakey II or III aortic dissection & $41.7 \%(5 / 12)$ \\
Stroke within 30 d & $25.0 \%(3 / 12)$ \\
Extreme hemodynamic compromise requiring & $25.0 \%(3 / 12)$ \\
$\quad$ CPR & \\
Connective tissue disorder & $8.3 \%(1 / 12)$ \\
Arch or proximal descending aorta $>45 \mathrm{~mm}$ & $0 \%(0 / 12)$ \\
\hline
\end{tabular}

$C P R$, Cardiopulmonary resuscitation

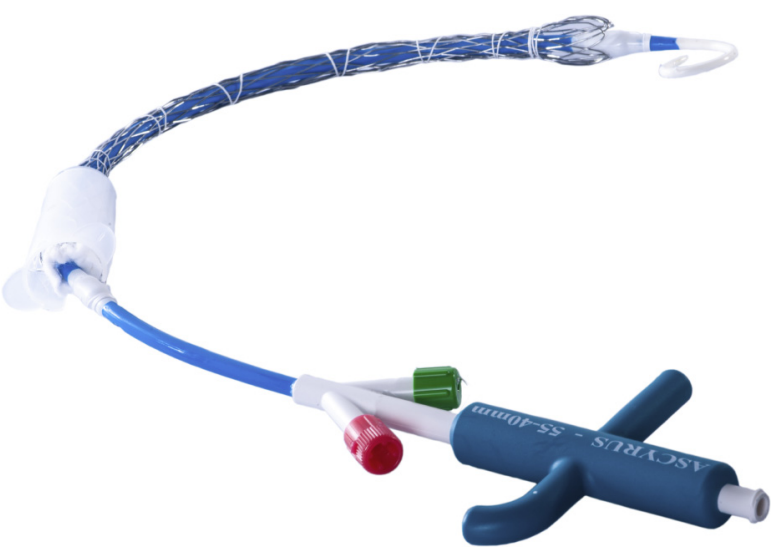

FIGURE 1. The Ascyrus Medical Dissection Stent contained within its delivery system.

within the aorta. The self-expandable device is available in 4 sizes: AMDS 40- to 30-mm tapered, AMDS 40-mm, AMDS 50- to 40-mm tapered, and AMDS 50-mm. The deployed length is dependent on the aortic diameter. The unit length at minimum and maximum instructions for use diameter is, respectively, for the AMDS 40- to 30-mm tapered, $210 \mathrm{~mm}$ and $170 \mathrm{~mm}$; for the AMDS 40-mm, $208 \mathrm{~mm}$ and $155 \mathrm{~mm}$; for the AMDS 50- to 40-mm tapered, $225 \mathrm{~mm}$ and $190 \mathrm{~mm}$; and for the AMDS 50-mm, $231 \mathrm{~mm}$ and $195 \mathrm{~mm}$.

\section{Device Sizing and Implantation}

The AMDS size is selected on the basis of the preprocedural MSCT. Two or 3-dimensional views can be used and the aortic diameter at the level between the innominate and left common carotid arteries (proximal diameter) and the aortic diameter at the level of T6 or pulmonary artery bifurcation (distal diameter) is measured. As such, an AMDS 40- to 30-mm tapered device fits within a proximal diameter of 20 to $35 \mathrm{~mm}$ and a distal diameter of 20 to $24 \mathrm{~mm}$, an AMDS 40-mm device fits within a proximal diameter of 20- to 35-mm and a distal diameter of 25- to 35-mm, an AMDS 50- to 40-mm tapered device fits within a proximal diameter of 36 to $45 \mathrm{~mm}$ and a distal diameter of 27 to $35 \mathrm{~mm}$, and an AMDS 50-mm device fits within a proximal diameter of 36 to $45 \mathrm{~mm}$ and a distal diameter of 36 to $45 \mathrm{~mm}$. The sizing system is simple, typically performed by the implanting surgeon upon reviewing the initial admission MSCT scan.

The implantation of the AMDS is performed as part of the standard of care hemiarch aortic reconstruction (Figures 3 and 4). Antegrade cerebral perfusion can be performed by cannulating the axillary artery, or by cautious direct cannulation of the arch branch vessel ostia being vigilant to minimize manipulation and creation of iatrogenic tears in the intimal flap. When circulatory arrest has been established, the ascending aorta is transected and resected. It is recommended to leave at least $1.0 \mathrm{~cm}$ of aortic tissue proximal to the takeoff of the innominate trunk to avoid obstruction of the innominate trunk by the polytetrafluoroethylene (PTFE) cuff. The lesser curvature should be left slightly longer than is customary with a hemiarch procedure to facilitate the AMDS implantation. The chosen AMDS size is now flushed and soaked with saline and inserted directly into the TL under direct vision until the entire device is inside the TL and the edge of the PTFE cuff is flush with the edge of the transected aorta. The protective sheath covering the PTFE cuff is removed and the PTFE cuff is expanded. Four interrupted 3-0 sutures are used to attach the PTFE cuff of the AMDS to the aorta using a PTFE felt strip external to the aorta to buttress the friable tissue, essentially sandwiching the location of the hemiarch anastomosis. The remainder of the hemiarch reconstruction is performed in the routine fashion securing the appropriately sized polyester 


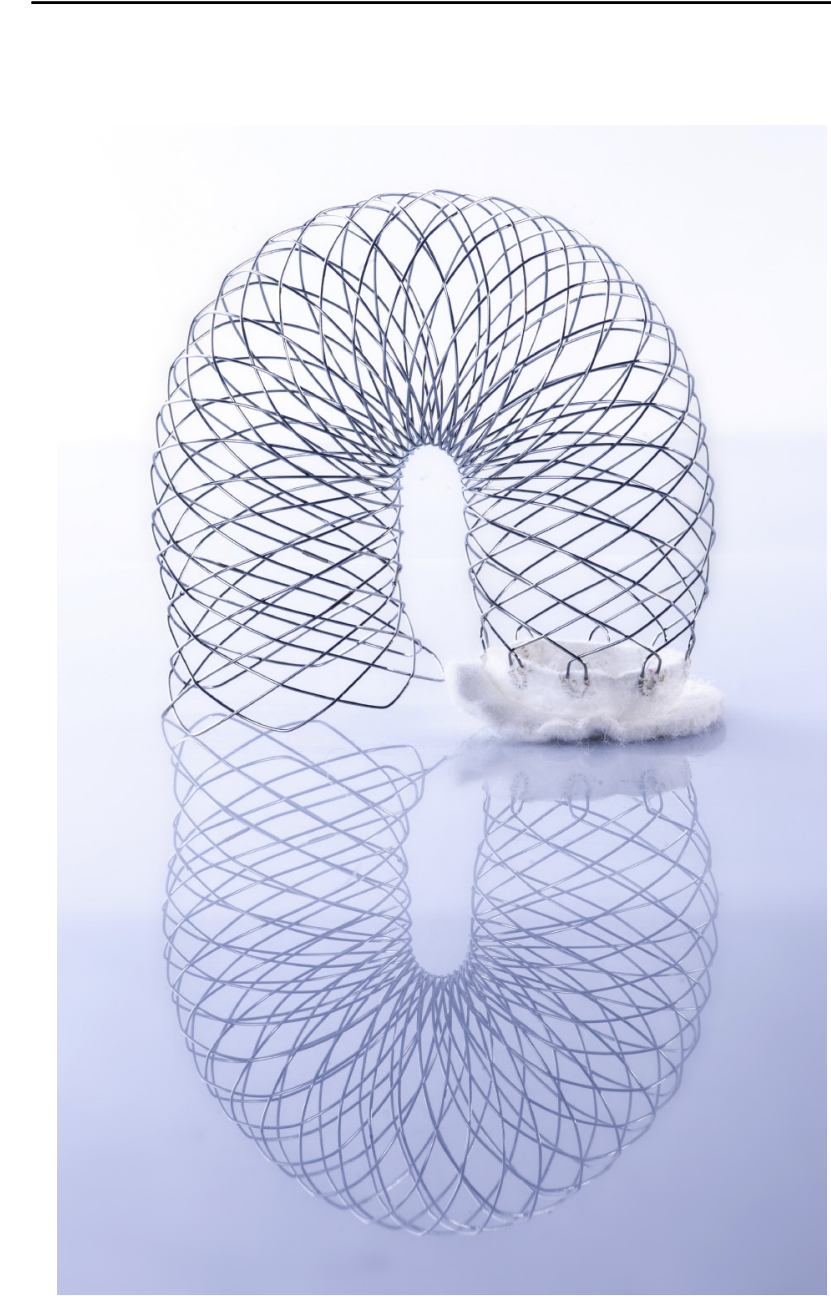

FIGURE 2. The fully expanded Ascyrus Medical Dissection Stent outside of its delivery system.

graft to the newly reconstructed aortic arch. Although the AMDS can be used with a guide wire and under fluoroscopy, in this series the AMDS was implanted directly without the use of $\mathrm{x}$-ray imaging or other adjuncts (Video 1).

\section{Statistical Analysis}

Continuous variables are expressed as mean \pm standard deviation and categorical variables are expressed as n $(\%)$. Data analyses were performed using Microsoft Excel version 16.11.1 (Microsoft, Redmond, Wash).

\section{RESULTS}

\section{Baseline Characteristics}

The baseline clinical characteristics as well as technical implantation data are shown in Table 1. Mean age was $66 \pm 12$ years and $6(37.5 \%)$ patients were female. Mean follow-up of $130 \pm 94$ days was obtained. A primary entry tear in the ascending aorta was identified in all 16 patients. One $(6.3 \%)$ patient required total arch replacement whereas $15(93.7 \%)$ underwent hemiarch repair. All patients $(\mathrm{n}=16)$ included in the study underwent arterial cannulation via the right axillary artery using an 8-mm Dacron graft sewn in an end-to-side fashion. Evidence of malperfusion

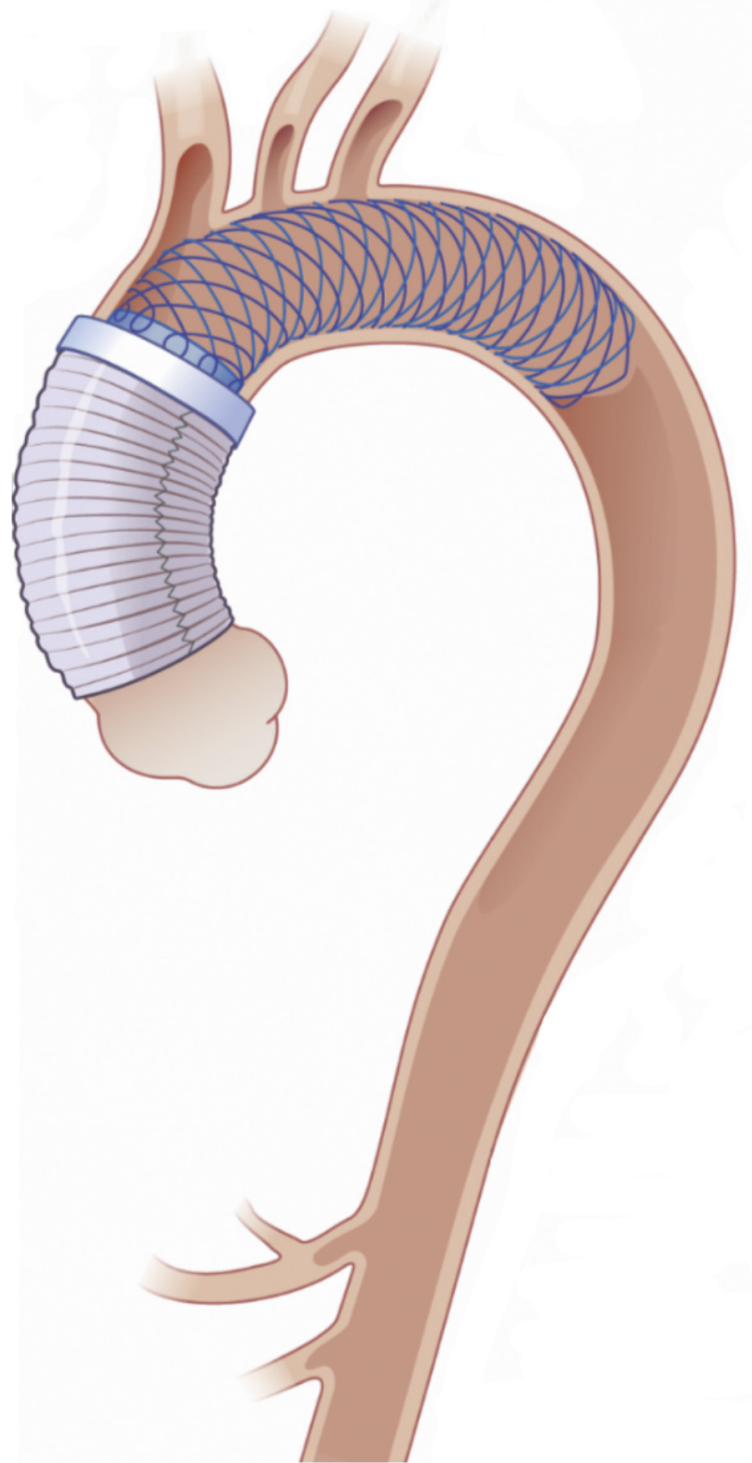

FIGURE 3. Illustration outlining the hemiarch anastomosis relative to the proximal cuff.

was present in $8(50 \%)$ of patients with $2(12.5 \%)$ having undergone previous sternotomy. Hypertension was present in $11(68.8 \%)$, chronic obstructive pulmonary disease in 3 $(18.8 \%)$, chronic renal failure in $2(12.5 \%)$, and previous stroke in $2(12.5 \%)$ patients. Mean deep hypothermic circulatory arrest duration was $33 \pm 12$ minutes with a mean AMDS implantation time of 2.1 minutes. Antegrade cerebral perfusion was used in all 16 patients with a mean cerebral perfusion duration of $27.3 \pm 10.4$ minutes. Postoperative anticoagulation strategy was available in 13 $(81.3 \%)$ patients. Nine patients received acetylsalicylic acid (ASA) $81 \mathrm{mg}$ daily alone, 1 received clopidogrel $75 \mathrm{mg}$ daily because of an ASA allergy, 1 received apixaban $5 \mathrm{mg}$ twice per day alone for concomitant atrial fibrillation, 


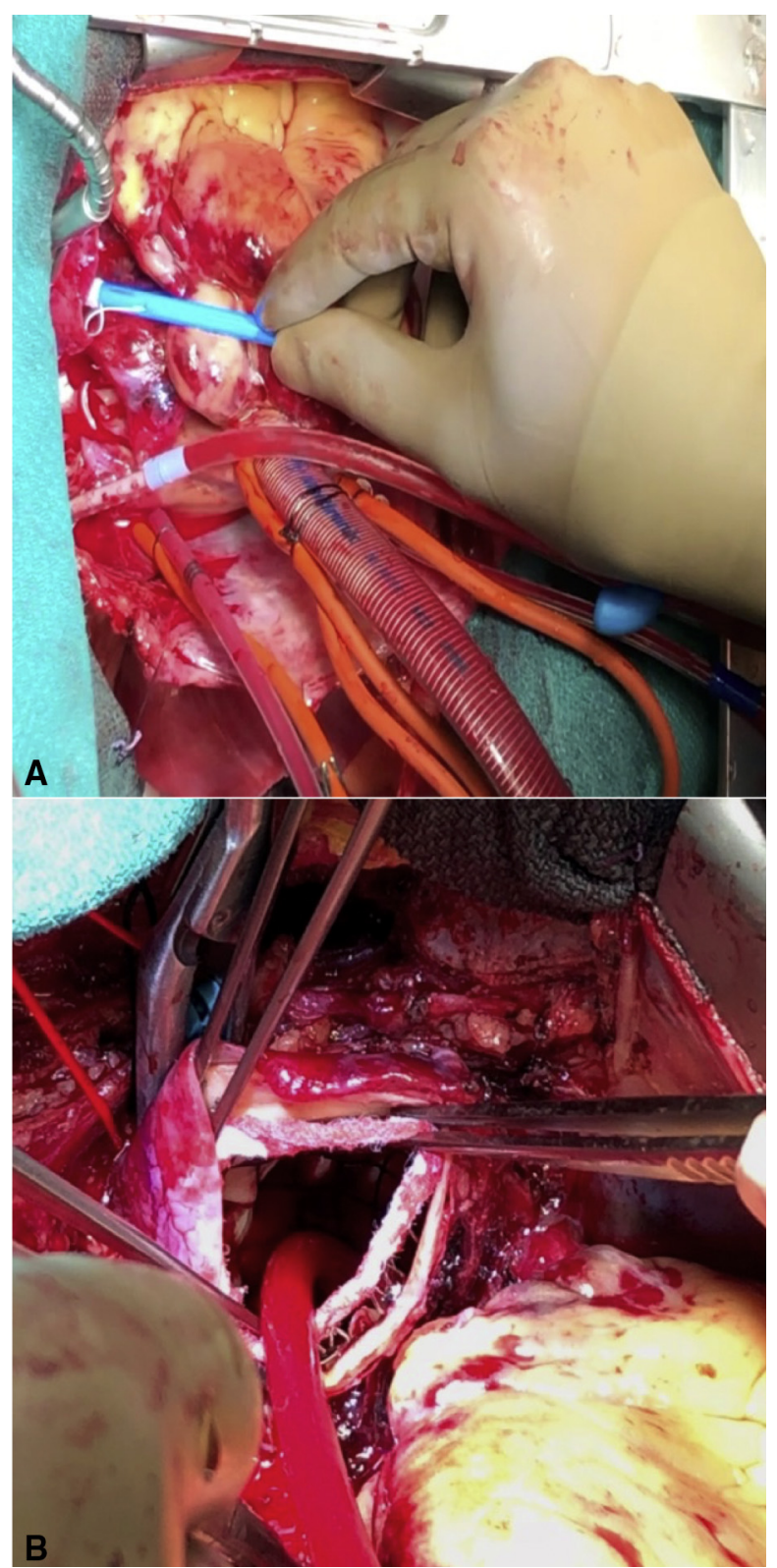

FIGURE 4. Intraoperative image of antegrade insertion of the Ascyrus Medical Dissection Stent at time of initial surgical repair (A) and fully deployed Ascyrus Medical Dissection Stent with proximal felt sewing cuff visible (B).

1 received ASA $81 \mathrm{mg}$ daily with warfarin for atrial fibrillation, and 1 received ASA $81 \mathrm{mg}$ daily with apixaban $5 \mathrm{mg}$ twice per day for atrial fibrillation.

\section{Safety Outcomes}

The procedural-related complications and hospital course of the study population are shown in Table 3 . Surgical repair of acute DeBakey I aortic dissection with AMDS implantation was successfully performed in all patients. At 30 days, $1(6.3 \%)$ mortality and $1(6.3 \%)$ neurological deficit occurred. There were no

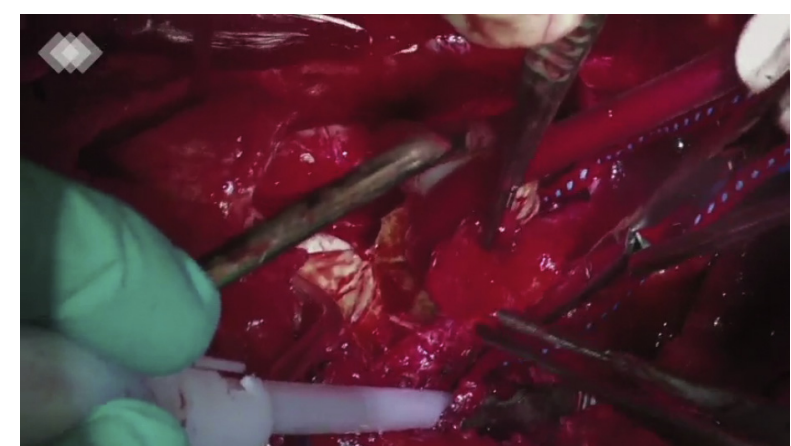

VIDEO 1. Intraoperative demonstrative video outlining the AMDS insertion technique. Video available at: https://www.jtcvs.org/article/S00225223(18)32828-9/fulltext.

device-related reinterventions or aortic injury associated with device implantation, and aortic branch vessel patency was $100 \%$ postimplantation. At mean follow-up of $130 \pm 94$ days, there was only 1 subsequent intervention that was a successful left renal artery stenting to treat static malperfusion. Median intensive care unit length of stay was 5 days interquartile range, 3-10 days), whereas median hospital length of stay was 15 days (interquartile range, 12-29 days). Acute renal failure, defined as a creatinine greater than double of baseline occurred in $8(50 \%)$ patients, whereas none required dialysis. Three $(18.8 \%)$ patients experienced respiratory failure and $1(6.3 \%)$ required temporary mechanical circulatory support for 11 days with subsequent device explantation. No device-related mortality or complications have been observed during the follow-up period.

\section{Performance Outcomes}

The performance-related outcomes of the study population are shown in Tables 4 and 5 and relate primarily to analyzing the success of malperfusion treatment and for the presence of positive aortic remodeling, as previously defined. During the follow-up period, 12 (75\%) patients had completed at least 1 postoperative MSCT scan (Figure 5). At initial follow-up MSCT scan, positive remodeling and complete or partial FL thrombosis of the aortic arch occurred in $11(91.7 \%)$ cases. Similarly, in the proximal descending thoracic aorta, positive remodeling and complete or partial FL thrombosis occurred in 11 $(91.7 \%)$ cases. All cases with dynamic malperfusion, including superior mesenteric artery malperfusion $(\mathrm{n}=2)$ and stenosis/occlusion of the proximal supraaortic vessels showed perfusion and recovery of the involved vessels $(n=4)$. Two patients with preimplantation acute lower extremity paralysis showed full postoperative recovery of lower extremity function. Two patients with no renal perfusion on the preimplantation MSCT scan showed enhancement and perfusion of the kidneys on the first postoperative MSCT scan. 
TABLE 3. Safety outcomes for the study population

\begin{tabular}{lc}
\hline \multicolumn{1}{c}{ Safety outcomes } & AMDS device $(\mathbf{n}=\mathbf{1 6})$ \\
\hline Successful device deployment & $100 \%(16 / 16)$ \\
30-Day mortality & $6.3 \%(1 / 16)$ \\
\hline $\begin{array}{l}\text { Neurologic deficit } \\
\text { Aortic injury associated with device } \\
\text { implantation }\end{array}$ & $6.3 \%(1 / 16)$ \\
Aortic arch branch vessel patency & $0 \%(0 / 16)$ \\
Device-related reintervention & $100 \%(0 / 16)$ \\
Disease-related reintervention* & $0 \%(0 / 16)$ \\
\hline $\begin{array}{l}\text { AMDS, Ascyrus Medical Dissection Stent. *One patient underwent successful left } \\
\text { renal artery stenting for distal malperfusion. }\end{array}$ & $6.3 \%(1 / 16)$ \\
\hline
\end{tabular}

\section{DISCUSSION}

Hemiarch aortic reconstruction for acute DeBakey I aortic dissection, despite being the standard of care approach, leaves residual untreated FL with patency rates of $69.7 \%$ to $78 \%$ at follow-up. ${ }^{1-4}$ This results in unfavorable remodeling occurring in $76 \%$ of patients undergoing hemiarch reconstruction. ${ }^{5}$ Several experts have advocated for an extended aortic repair performing a prophylactic total arch replacement with or without adding a stent graft implant into the TL of the descending aorta to remove and exclude any possible re-entry tears in the arch and the proximal descending aorta, the so-called frozen elephant trunk (FET) technique. Although this technique is a sensible approach to elective complex arch aneurysms, when applied to treatment of acute dissections it will extend an already challenging operation in gravely ill patients, increase the time on cardiopulmonary bypass, circulatory arrest, and selective cerebral perfusion. ${ }^{15}$ The FET also exposes the patient to additional risks of stroke, bleeding, and paralysis due to the extended operation requiring the resection and replacement of the aortic arch and the supra-aortic branches and the obstruction of the blood supply to the spinal cord if the FET coverage is more than $10 \mathrm{~cm} .{ }^{16}$ To date no prospective controlled trial has assessed the efficacy of using a short FET in the descending aorta and most publications have been on the basis of the experiences of individual institutions with a great interest in the FET technique. The concept of an emergency prophylactic

TABLE 4. Performance outcomes for the study population

\begin{tabular}{lc}
\hline \multicolumn{1}{c}{ Performance outcome } & $\begin{array}{c}\text { AMDS device } \\
(\mathbf{n}=\mathbf{1 2})^{*}\end{array}$ \\
\hline $\begin{array}{l}\text { Positive remodeling with or without complete } \\
\text { or partial FL thrombosis of aortic arch }\end{array}$ & $91.7 \%(11 / 12)$ \\
Positive remodeling with or without complete & $91.7 \%(11 / 12)$ \\
or partial FL thrombosis of proximal \\
descending thoracic aorta
\end{tabular}

complex arch replacement to avoid chronic complications of the arch and descending aorta is also controversial. The inability to predict the patients who would require secondary operations after the index emergency operation leaves many of these vulnerable patients exposed to complex emergency prophylactic arch replacement and its inherent risks to avoid possible future clinical arch complications and redo operations in an unknown patient group. Additionally, no controlled study has verified the potential benefits of the FET in an emergency dissection setting. The FET might be a useful approach in acute dissections where the arch is aneurysmal, an entry tear is identified within the arch proper when the overall condition of the patient allows for a more prolonged, complex procedure, or where there is rupture of the descending aorta associated with dissection. ${ }^{17}$

We believe contemporary modern treatment of acute DeBakey I dissections requires a dissection-specific therapy to address the unique challenges with as few as possible additional risks to this extremely compromised patient group. In our opinion, the ideal hybrid graft for treatment of this devastating disease should have low complication rates, be easy to implant, not significantly add to the operative time, not add to the bleeding or spinal cord injury risk, be able to induce positive remodeling, and treat dynamic malperfusions in the setting of the index operation.

As shown by Rylski and colleagues, ${ }^{5}$ in $>70 \%$ of the cases, even after "successful" hemiarch reconstruction, a proximal tear can be found at the site of the distal graft to aorta anastomosis and this entry tear can be found in $96 \%$ of the patients who show accelerated aortic growth. The additional re-entry tears with or without contribution of other distal re-entry tears that might act as venting mechanisms could contribute to the flow into and the pressurization of the FL above a critical threshold, inducing negative remodeling. The intimal tear at the distal hemiarch graft to aorta anastomosis allows for a significant APF within the FL that can be readily detected with echocardiography and CT scans. The APF pressurizes the FL, increasing the wall tension in the weakened aortic wall (La Place's law), collapsing the TL thus further increasing the flow and pressure in the FL causing malperfusion and/or negative remodeling. One method of slowing or reversing this process and achieving positive remodeling is to close the proximal-most communications with the FL. Additionally, by expanding and supporting the collapsed TL, the blood flow is preferentially channeled from the ascending aorta to the TL pressurizing the TL. Reduction of the flow and pressure in the FL below the critical remodeling threshold causes collapse and complete or partial thrombosis of the FL. The flow reduction in the FL in combination with pressurization and support of the TL can encourage induction of positive remodeling in addition to improving end-organ blood flow and malperfusion. Obliteration of the FL, in addition 
TABLE 5. Summary of aortic remodeling and false lumen status post-AMDS insertion

\begin{tabular}{|c|c|c|c|c|c|c|c|c|c|c|c|c|c|}
\hline \multirow[b]{2}{*}{ Subject $(n=12)^{*}$} & \multirow[b]{2}{*}{ Follow-up } & \multicolumn{6}{|c|}{ Remodeling $\dagger$} & \multicolumn{6}{|c|}{ FL Status } \\
\hline & & Arch & PDA & MDA & DDA & Paravisceral & $\overline{\mathbf{A A}}$ & Arch & PDA & MDA & DDA & Paravisceral & $\overline{\mathbf{A A}}$ \\
\hline 001 & 6 Months & + & + & + & + & + & $\leftrightarrow$ & Obl & Obl & Obl & $\mathrm{PT}$ & PT & $\mathrm{P}$ \\
\hline 002 & 6 Months & + & + & N/A & N/A & N/A & N/A & Obl & Obl & N/A & N/A & N/A & N/A \\
\hline 003 & 6 Months & + & + & N/A & N/A & N/A & N/A & Obl & Obl & N/A & N/A & N/A & N/A \\
\hline 004 & 3 Months & + & + & + & + & + & $\leftrightarrow$ & Obl & Obl & $\mathrm{P}$ & $\mathrm{P}$ & $\mathrm{P}$ & $\mathrm{P}$ \\
\hline 005 & 1 Month & N/A & $\leftrightarrow$ & + & + & + & + & N/A & $\mathrm{T}$ & $\mathrm{T}$ & $\mathrm{P}$ & $\mathrm{P}$ & $\mathrm{P}$ \\
\hline 006 & Postoperative & + & + & + & + & $\leftrightarrow$ & $\leftrightarrow$ & Obl & PT & PT & $\mathrm{P}$ & $\mathrm{P}$ & $P$ \\
\hline 007 & Postoperative & + & + & + & + & $\leftrightarrow$ & $\leftrightarrow$ & PT & PT & PT & $\mathrm{T}$ & $\mathrm{P}$ & $\mathrm{P}$ \\
\hline 008 & Postoperative & + & $\leftrightarrow$ & + & + & + & N/A & Obl & PT & $\mathrm{P}$ & PT & PT & N/A \\
\hline 009 & Postoperative & + & + & + & + & + & N/A & Obl & $\mathrm{P}$ & $\mathrm{P}$ & $\mathrm{P}$ & $\mathrm{P}$ & N/A \\
\hline 010 & 1 Month & + & + & N/A & N/A & N/A & N/A & Obl & Obl & N/A & N/A & N/A & N/A \\
\hline 011 & 1 Month & + & $\leftrightarrow$ & + & + & $\leftrightarrow$ & N/A & PT & PT & PT & $\mathrm{P}$ & $\mathrm{P}$ & N/A \\
\hline 012 & Postoperative & + & + & + & + & + & + & $\mathrm{P}$ & $\mathrm{P}$ & $\mathrm{P}$ & $\mathrm{P}$ & $\mathrm{P}$ & $\mathrm{P}$ \\
\hline
\end{tabular}

$P D A$, Proximal descending aorta; $M D A$, mid-descending aorta; $D D A$, distal descending aorta; Paravisceral, paravisceral aorta; $A A$, abdominal aorta; $O b l$, obliterated; $P T$, partial thrombosis; $P$, patent; $N / A$, not applicable; $T$, complete thrombosis. *Computed tomography scans were completed for $12 / 16$ patients during the follow-up period. $\dagger$ “ + " indicates positive remodeling, and " $\leftrightarrow$ ” indicates no change.

to complete and partial thrombosis of the AMDS-treated FL serve as surrogates for reduction of flow and pressure within the FL.

On the basis of the scientific background and the current state of the art discussed previously, the need for a dissection-specific therapy was identified. The attributes sought in this dissection-specific therapy included:

1. Ability to effectively abolish the APF;

2. Support and expand the intimal flap and the TL;

3. By expanding the intimal flap, expose any potential downstream re-entry tears to allow for minimally invasive approaches to entry tears and the FL rather than risky, invasive "total aorta" resections;

4. Long enough coverage to alleviate malperfusion distally without increasing the risk for paralysis;

5. Not require additional material and staff support to suit the needs of a true emergency condition;

6. Not add any significant time to the standard hemiarch repair to reduce additional morbidity and mortality;

7. Ability to be used by cardiac surgeons with or without special interest in aortic surgery.

In this article, we report the initial Canadian experience with the use of a dissection-specific device, the AMDS, as an adjunct to conventional standard of care surgical repair of acute DeBakey I aortic dissection. The primary mechanism through which the AMDS promotes positive remodeling is by abolishing pulsatile antegrade flow into the FL and expanding and supporting the TL (Graphical Abstract). Procedural success was achieved in all patients without major periprocedural complications and with a 30-day mortality of $6.3 \%$. Notably, there were excellent rates of positive aortic remodeling in the aortic arch $(91.7 \%)$ and proximal descending thoracic aorta $(91.7 \%)$. This report describes the early results of the DARTS trial and as such the remodeling data have been captured as 2-dimensional data points measuring the diameter of the TL and the largest depth of the FL at all measuring points identified on CT angiography. As shown by Sobocinski and colleagues, ${ }^{18}$ another method of analyzing aortic remodeling is using volume analysis of the TL and FL. The authors intend to perform such analyses at the completion of the DARTS trial.

Furthermore, the AMDS manages dynamic malperfusion in the setting of acute DeBakey I aortic dissection presentation with favorable recovery of the involved vessels in the thoracic and abdominal aorta, including proximal supraaortic stenosis/occlusion, visceral vessels, and lower extremity. The 2 cases of complete paralysis highlight, to our knowledge, for the first time, that neurological recovery from malperfusion is possible. To date, no device-related mortality or complications with the AMDS have been observed attesting to the safety of the device. Additional use of the AMDS with the standard aortic repair technique allows treatment of the FL and malperfusion at the time of initial surgery with a high rate of positive remodeling in the aorta. This represents a significant improvement over standard hemiarch aortic reconstruction alone, where the untreated FL contributes to malperfusion and unfavorable remodeling in most patients, ultimately leading to further intervention and mortality. ${ }^{6,7}$

Patients with connective tissue disorders were excluded in this study to avoid confounding the initial safety and performance trial data. The AMDS is designed with a chronic expansion force at approximately $20 \%$ of the normal tensile 


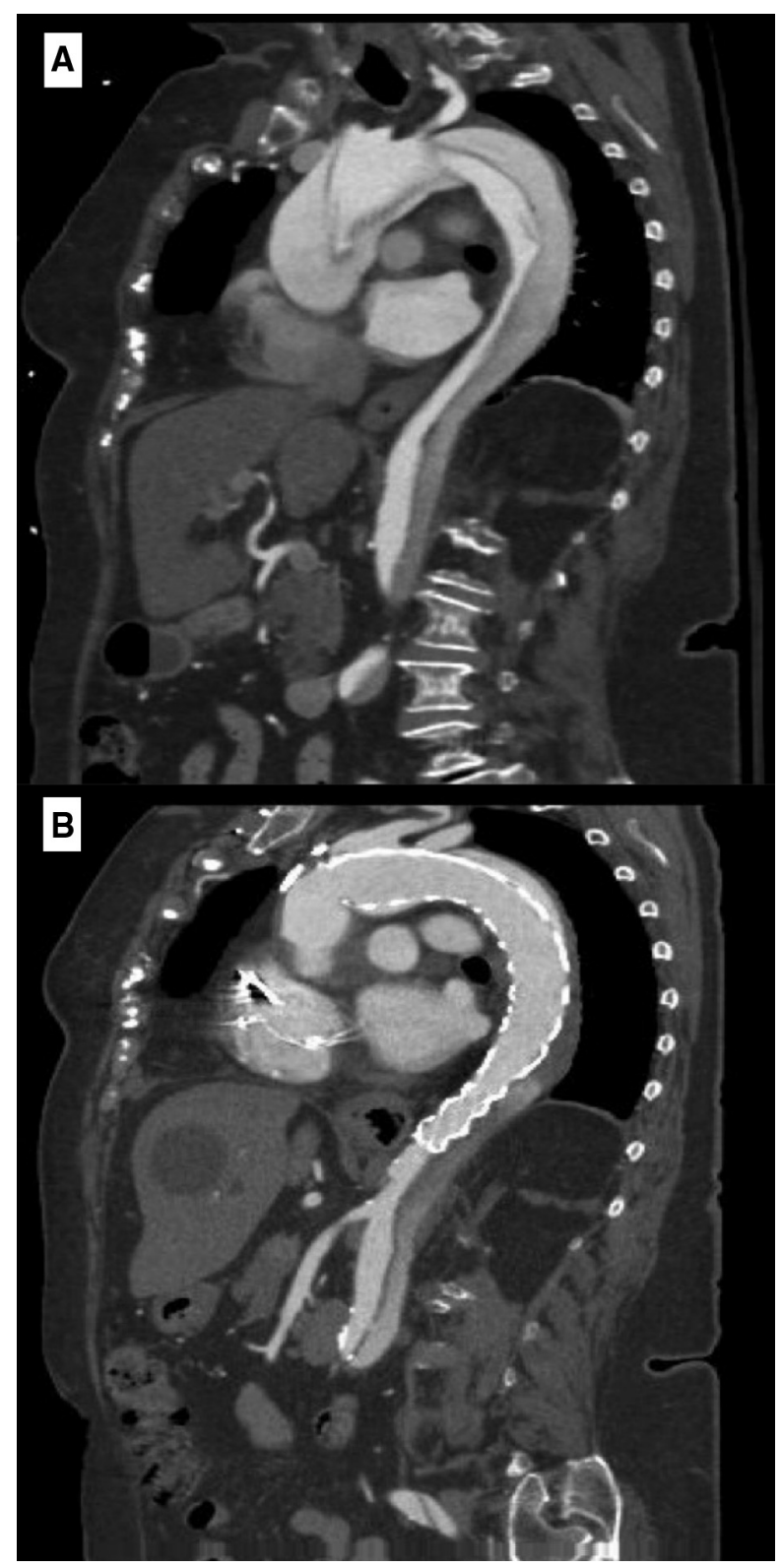

FIGURE 5. Preoperative multislice computed tomography (A) showing acute aortic dissection and postoperative multislice computed tomography (B) showing the deployed Ascyrus Medical Dissection Stent.

strength of the aortic wall, thus it exerts low and gentle pressure on the aorta. Additionally, finite element analysis studies of the AMDS show the distribution of forces and strain across the Nitinol frame to be uniform and low. Thus, as opposed to the typical Z- or M-ring stent design where the forces vary in the different areas of the wire form and can cause injury to the aortic wall or the intimal flap of a dissection, the low and uniform strain and forces of the AMDS were designed to avoid such complications. This design might be appropriate for treatment of patients with connective tissue disorders, but an appropriately designed and structured study will need to address this question.

\section{Limitation}

The main limitation lies with the small number of patients and the nonrandomized, single-armed nature of the study. No comparison with standard surgical repair alone performed at these centers is provided. Further recruitment and ongoing follow-up are thus required to further evaluate the clinical outcomes with the AMDS device.

\section{CONCLUSIONS}

The present initial Canadian results of the DARTS trial suggest that the AMDS is a safe, feasible, and reproducible adjunct to current surgical approaches for acute DeBakey I aortic dissection repair without prolonging the procedure. Furthermore, the AMDS promotes early positive remodeling in the aortic arch and distal dissected segments, with favorable rates of positive remodeling at follow-up. Finally, because of its long thoracic aortic coverage, the AMDS is an effective treatment for dynamic malperfusion involving major aortic branches. Ongoing follow-up and continued enrollment will provide additional insight into the longterm effects of the AMDS on overall aortic remodeling and patient outcomes.

\section{Conflict of Interest Statement}

Dr Chu is a speaker for Medtronic, LivaNova, Abbott Vascular, and Boston Scientific. Dr Shahriari is President and shareholder for Ascyrus Medical LLC. All other authors have nothing to disclose with regard to commercial support.

\section{References}

1. Moore NR, Parry AJ, Trottman-Dickenson B, Pillai R, Westaby S. Fate of the native aorta after repair of acute type A dissection: a magnetic resonance imaging study. Heart. 1996;75:62-6.

2. Sultan I, Wallen TJ, Habertheuer A, Siki M, Arnaoutakis GJ, Bavaria J, et al. Concomitant antegrade stent grafting of the descending thoracic aorta during transverse hemiarch reconstruction for acute DeBakey I aortic dissection repair improves aortic remodeling. J Card Surg. 2017;32:581-92.

3. Park K, Lim C, Choi JH, Chung E, Choi SI, Chun EJ, et al. Midterm change of descending aortic false lumen after repair of acute type I dissection. Ann Thorac Surg. 2009;87:103-8.

4. Fattori R, Bacchi-Reggiani L, Bertaccini P, Napoli G, Fusco F, Longo M, et al. Evolution of aortic dissection after surgical repair. Am J Cardiol. 2000;86: 868-72.

5. Rylski B, Hahn N, Beyersdorf F, Kondov S, Wolkewitz M, Blanke P, et al. Fate of the dissected aortic arch after ascending replacement in type A aortic dissection. Eur J Cardiothorac Surg. 2017;51:1127-34.

6. Evangelista A, Salas A, Ribera A, Ferreira-González I, Cuellar H, Pineda V, et al. Long-term outcome of aortic dissection with patent false lumen: predictive role of entry tear size and location. Circulation. 2012;125:3133-41.

7. Kobuch R, Hilker M, Rupprecht L, Hirt S, Keyser A, Puehler T, et al. Late reoperations after repaired acute type A aortic dissection. J Thorac Cardiovasc Surg. 2012;144:300-7.

8. Malvindi PG, van Putte BP, Sonker U, Heijmen RH, Schepens MA, Morshuis WJ. Reoperation after acute type a aortic dissection repair: a series of 104 patients. Ann Thorac Surg. 2013;95:922-7. 
9. Olsson C, Hillebrant C, Liska J, Lockowandt U, Eriksson P, Franco-Cereceda A. Mortality and reoperations in survivors operated on for acute type A aortic dissection and implications for catheter-based or hybrid interventions. J Vasc Surg. 2013;58:333-9.e1.

10. Czerny M, Stöhr S, Aymard T, Sodeck GH, Ehrlich M, Dziodzio T, et al. Effect on false-lumen status of a combined vascular and endovascular approach for the treatment of acute type A aortic dissection. Eur J Cardiothorac Surg. 2012;41: 409-13.

11. Lombardi JV, Cambria RP, Nienaber CA, Chiesa R, Teebken O, Lee A, et al. Prospective multicenter clinical trial (STABLE) on the endovascular treatment of complicated type B aortic dissection using a composite device design. $J$ Vasc Surg. 2012;55:629-40.e2.

12. Brunkwall J, Kasprzak P, Verhoeven E, Heijmen R, Taylor P. Endovascular repair of acute uncomplicated aortic type B dissection promotes aortic remodelling: 1 year results of the ADSORB trial. Eur J Vasc Endovasc Surg. 2014;48: 285-91.

13. Bavaria JE, Brinkman WT, Hughes GC, Khoynezhad A, Szeto WY, Azizzadeh A, et al. Outcomes of thoracic endovascular aortic repair in acute type B aortic dissection: results from the valiant United States investigational device exemption study. Ann Thorac Surg. 2015;100:802-8; discussion: 808-9.
14. Tsai M, Wu H, Roan J, Tsai YS, Hsieh PCH, Yang YJ, et al. Effect of false lumen partial thrombosis on repaired acute type A aortic dissection. $J$ Thorac Cardiovasc Surg. 2014;148:2140-6.e3.

15. Bozso SJ, White A, Nagendran J, Moon MC, Chu MW. Hybrid aortic arch and frozen elephant trunk reconstruction: bridging the gap between conventional and total endovascular arch repair. Expert Rev Cardiovasc Ther. 2018; 16:209-17.

16. Shrestha M, Martens A, Kaufeld T, Beckmann E, Bertele S, Krueger H, et al. Single-centre experience with the frozen elephant trunk technique in 251 patient over 15 years. Eur J Cardiothorac Surg. 2017;52:858-66.

17. Shrestha M, Bachet J, Bavaria J, Carrel TP, De Paulis R, Di Bartolomeo R, et al Current status and recommendations for use of the frozen elephant trunk technique: a position paper by the vascular domain of EACTS. Eur J Cardiothorac Surg. 2015;47:759-69.

18. Sobocinski J, Lombardi JV, Dias NV, Berger L, Zhou Q, Jia F, et al. Volume analysis of true and false lumens in acute complicated type B aortic dissections after thoracic endovascular aortic repair with stent grafts alone or with a composite device design. J Vasc Surg. 2016;63:1216-24.

Key Words: aortic dissection, endovascular 